\title{
Ionization features in cathode spot region of vacuum arc
}

A.V. Ushakov ${ }^{a, b^{*}}$, I.V. Karpov ${ }^{b}$, A.A. Shaihadinov ${ }^{b}$, L.Yu. Fedorov ${ }^{a, b}$, E.A. Goncharova ${ }^{b}$, V.G. Demin ${ }^{b}$

${ }^{a}$ Federal Research Center Krasnoyarsk Scientific Center of the Siberian Branch of the Russian Academy of Sciences, 660036, Krasnoyarsk, Russia

${ }^{b}$ Siberian Federal University, 660041, Krasnoyarsk, Russia

E-mail address: ushackov@mail.ru (A.V. Ushakov)

\begin{abstract}
A model of ionization processes in a low-temperature plasma within the cathode region of a vacuum arc is presented. This mathematical model is represented by a system of nonlinear differential equations of drift-diffusion for the electron density and the average energy of second-order electrons. The system of equations was solved by the finite element method in two coordinates in numerical form using the COMSOL Multiphysics software program. It was shown that due to nonequilibrium processes, Langmuir waves appeared at the plasma boundary, which leads to multistage ionization of metal vapor. The arc discharge was presented in the form of a cylindrical plasma column with a length of $200 \mu \mathrm{m}$ and a diameter of $50 \mu \mathrm{m}$. At the interface between the cathode and the plasma column, a double electric layer has appeared which holds hot electrons in the central zone of the discharge. High specific power was focused close to the cathode surface, which provided the continuous supply of metal vapor. The numerical results of the model have appeared to be in good agreement with the experimental data.
\end{abstract}

Keywords: mathematical modeling, vacuum arc, cathode spot, ionization, Langmuir waves.

\section{Introduction}

A vacuum arc discharge is a complex phenomenon that includes physicochemical processes at the electrodes and interelectrode space. The main parameters that determine the functioning of the cathode spot of a vacuum arc include the ratio of the densities of ion plasma and electron emission currents, the electric field strength in the cathode region due to the space charge of ions, the electron and ion temperature in the cathode plasma, the temperature of the melt surface in a microcrater, etc. $[1,2]$. At present, two basic ideas have been formed on the nature of the cathode spot: the first model assumes the entry of electrons into the plasma due to thermal and field emission, and the heating of the spot due to ion bombardment [3, 4]. In the second model, the emission of electrons and Joule heating is carried out due to the explosion of micro-tips $[5,6]$. However, the question of the mechanisms 
of ionization in the cathode plasma, the processes of formation of the space charge of Langmuir remains open. In [7, 8], the results of experimental studies were published showing the relationship between the processes in the cathode plasma and the properties of the synthesized nanoparticles. Based on the experiments, it was concluded that all ionization processes proceed similarly to a spark discharge.

However, the efficiency of ionization, in the case of a small length of this region and low potential, remains undecided. In this regard, it is necessary to analyze the experimental results, obtained during the study of the threshold intensity of metal vapors breakdown in the cathode spot zone in a wide range of pressures, temperatures, and magnetic fields, and to understand the physical mechanisms related to ionization caused by various phenomena. In addition to the main condition $p d=$ constant, it is necessary to take into account several aspects of maintaining the discharge at submillimeter distances. The increase in pressure complicates the collision process and many reference values may be wrong. Under the conditions of intensive evaporation, it is necessary to take into account the pressure gradient, the formation of molecules and clusters, the complication of the plasma chemistry, and the change in the plasma frequency during ionization. Taking these important parameters into account can lead to a significant complication of the model.

In this work, for the theoretical analysis of the obtained data, a numerical model is used, based on the numerical solution of the drift-diffusion equation, which describes the change in the density of electrons and ions due to the mutual effect of the processes of multielectron, avalanche and step ionization, taking into account the recombination processes due to energy loss due to elastic collisions between an electron and a neutral vapor molecule; loss of electrons due to diffusion from the cathode spot zone.

The obtained results can be used to develop technologies for the synthesis of nanomaterials in plasma of a low-pressure arc discharge [9-12]. This pressure range is of great interest for technologies related to aerospace engineering, since the operation of equipment at low pressure is influenced by the electrical breakdowns. Besides, the nanosecond pulses used in this model are suitable for studying the role of ionization-related losses.

\section{Physical model}

Based on numerous experimental data, we can conclude that the physicochemical processes in the plasma of the cathode region of the vacuum arc proceed as follows. The cathode surface in the region of the cathode spot is heated to a boiling point, while the cathode material is intensively evaporated and the vapor pressure can reach 100 atm depending on the material. In our model, copper was selected as the cathode material and 
vapor pressure of $10 \mathrm{~atm}$ was adopted. The main potential drop within the interelectrode space occurs in a region measured as equal to the order of the diameter of the cathode spot $(200 \mu \mathrm{m})$. The ion cloud, generating the potential necessary to accelerate free electrons, is a cause of this effect. The electric field strength sufficient to initiate a small field current is created in the region of the main potential drop. The emitted electrons serve as seed electrons to excite the main discharge. Since the mobility of ions is 100 times less than the mobility of electrons, they do not participate in the ionization processes and, accordingly, the second and third Townsend coefficients can be neglected. Since the resulting plasma is nonequilibrium, Langmuir plasma oscillations arise, which determine ionization processes in the cathode region of the arc discharge. The energy generated as a result of high-frequency oscillations is spent on heating the steam and the surface of the cathode spot. Thus, the self-maintenance of arc discharge occurs. All the processes occur in the cathode spot for a characteristic time of 50 ns. If the plasma wave is plane, then the near-cathode plasma is described by one-dimensional equations. Quite different processes take place in the case of cyclotron waves.

\section{Model equations}

The electron density $n_{e}$ and the energy density $n_{\varepsilon}$ of electrons can be calculated by solving the system of temporal equations of drift-diffusion.

$$
\begin{gathered}
\frac{\partial}{\partial t}\left(n_{e}\right)+\nabla \cdot\left[-n_{e}\left(\boldsymbol{\mu}_{e} \times \mathbf{E}\right)-\mathbf{D}_{e} \times \nabla n_{e}\right]=R_{e} \\
\frac{\partial}{\partial t}\left(n_{\varepsilon}\right)+\nabla \cdot\left[-n_{\varepsilon}\left(\boldsymbol{\mu}_{\varepsilon} \times \mathbf{E}\right)-\mathbf{D}_{\varepsilon} \times \nabla n_{\varepsilon}\right]+\mathbf{E} \cdot \boldsymbol{\Gamma}_{e}=R_{\varepsilon}
\end{gathered}
$$

For the Maxwell distribution, using the Einstein's relations, we can calculate $\mathrm{D}_{e}$ (electron diffusion coefficient), $\mu_{\varepsilon}$ (mobility of energy), $\mathrm{D}_{\varepsilon}$ (energy diffusion coefficient) as a function of $\mu_{e}$ (mobility of electrons):

$$
\mathrm{D}_{e}=\mu_{e} T_{e}, \mu_{\varepsilon}=\frac{5}{3} \mu_{e}, \mathrm{D}_{\varepsilon}=\mu_{\varepsilon} T_{e}
$$

Since we are talking about plasma oscillations, separation of charges within the area of plasma shell is occurs due to a significant difference in mobility and diffusion between ions and electrons. This process generates high electric field strength, resulted in a significant increase in the average electron energy. Therefore, the above drift-diffusion equations are highly nonlinear. Within short distances, the electron density $n_{e}$ can vary by 10 orders of magnitude. Therefore, it is reasonable to solve the equations for the logarithm of electron density and energy. 
The coefficients for the electron source $R_{e}$ and the energy losses due to inelastic collisions $R_{\varepsilon}$ [13] in the above equations are determined by plasma chemistry. They are determined using a speed coefficient or Townsend coefficient. The inflow of electrons into the plasma within the cathode layer due to impact ionization is an energy-inefficient process. A stepwise process is more efficient. We will further assume that ionization in the cathode layer of a vacuum arc occurs in steps. For simplicity, we assume that ionization proceeds through one excited state. Such a state can be both the lower excited state of the $5 s^{2} S$ copper atom and the group of excited states [14]. Therefore, plasma chemistry is determined by the ratio: $\mathrm{Cu}^{*}+\mathrm{e}^{-} \rightarrow \mathrm{Cu}^{+}+2 \mathrm{e}^{-}$, where $*$ means an electron-excited state. For speed coefficients we can write the following relation:

$$
k_{k}=\gamma \int_{0}^{\infty} \varepsilon \sigma_{k}(\varepsilon) f(\varepsilon) d \varepsilon
$$

Integration is carried out concerning the electron energies $\varepsilon(\mathrm{V}), \gamma=\left(2 q / m_{e}\right)^{1 / 2}\left(\mathrm{C}^{1 / 2} / \mathrm{kg}^{1 / 2}\right), m_{e}$ is the electron mass $(\mathrm{kg}), \sigma_{k}$ is the collision cross-section $\left(\mathrm{m}^{2}\right)$, and $f$ is the energy distribution function of electrons. In our case, the Maxwell distribution is assumed. The collision crosssection is determined by plasma chemistry [14]. The index $k$, in this case, varies in the range, which depends on the type of reaction: $(0, M)$ range is used for the reactions that provide increase or decrease of electron density, $(0, P)$ range is used for the energy losses in inelastic collisions. In the case of stepwise ionization, it is assumed that $P \gg M$.

For an electron source, we can write the following expression

$$
R_{e}=\sum_{j=1}^{M} x_{j} k_{j} N_{n} n_{e}
$$

where $x_{j}$ is the molar fraction of particles involved in the reaction of $j$ with targets, and $N_{n}$ is the density of background gas $\left(1 / \mathrm{m}^{3}\right)$. The electron energy loss can be written as follows:

$$
R_{\varepsilon}=\sum_{j=1}^{P} x_{j} k_{j} N_{n} n_{e} \Delta \varepsilon_{j}
$$

where $\Delta \varepsilon_{j}(\mathrm{~V})$ is the energy loss as a result of reaction $j$.

For studying the mass transfer of heavy particles, the following drift-diffusion equation for a multicomponent mixture is solved:

$$
\rho \frac{\partial w_{k}}{\partial t}+\rho(\mathbf{u} \cdot \nabla) w_{k}=\nabla \cdot \mathbf{j}_{k}+R_{k}
$$


where $w_{k}$ is the mass fraction of the $k$-th species, $\mathbf{j}_{k}$ is the diffuse flux vector, $\mathbf{u}$ is the velocity vector averaged over the mass, $R_{k}, \mathrm{~kg} /\left(\mathrm{m}^{3} \mathrm{~s}\right)$, is the expression for the velocity for the $k$-th species. The equation is solved in the averaging approximation concerning a multicomponent mixture. In this model, instead of calculating the diffusion term $D_{i j}$ for a pair of each species of gas, only one averaged diffusion coefficient of the mixture is calculated [15]. For copper plasma near the cathode region, such an approximation will be more correct than the larger the mass of particles and when the electric field is large enough to ensure that the ion drift velocity is much higher than the thermal velocity of the ion. In this model, the ion drift velocity is proportional to the one that is often found in the experiments in the high-field limit. The averaged diffusion coefficient is calculated using the Einstein relation.

The electrostatic field is calculated using the Poisson equation:

$$
-\nabla \cdot \varepsilon_{0} \varepsilon_{r} \nabla V=\rho
$$

where $\varepsilon_{0}$ is the vacuum permittivity equal to $8.854 \cdot 10^{-12}(\mathrm{~F} / \mathrm{m}), \varepsilon_{r}$ is the relative permeability, $\rho$ is the density of bulk charge, calculated based on plasma chemistry by the formula:

$$
\rho=q\left(\sum_{k=1}^{N} Z_{k} n_{k}-n_{e}\right)
$$

where $Z_{k}$ is the ion charge.

Since the wavelength of plasma oscillations is less than the geometric dimensions of the plasma region, the high-frequency electric field is calculated using the following equation:

$$
\nabla \times\left(\mu_{r}^{-1} \nabla \times \mathbf{E}\right)-k_{0}^{2}\left(\varepsilon_{r}-\frac{j \sigma}{\omega \varepsilon_{0}}\right) \mathbf{E}=0
$$

where $\mu_{r}$ is the relative magnetic permeability, $k_{0}$ is the wave number in a vacuum, defined as $k_{0}=\omega \sqrt{\varepsilon_{0} \mu_{0}}=\frac{\omega}{c}$, and $c$ is the speed of light in vacuum.

In the presence of a magnetic field, the relationship between the density of plasma current $\mathbf{J}$ and the electric field $\mathbf{E}$ in the Ohm differential law becomes more complex:

$$
\sigma^{-1} \cdot \mathbf{J}=\mathbf{E}
$$

where $\sigma$ is the plasma conductivity, which is a tensor and a function of electron density, collision frequency, and magnetic flux density. 
In our model we deal with the diagonal inversion of plasma conductivity because there is no external constant magnetic field:

$$
\sigma^{-1}=\left[\begin{array}{ccc}
\frac{1}{\beta} & 0 & 0 \\
0 & \frac{1}{\beta} & 0 \\
0 & 0 & \frac{1}{\beta}
\end{array}\right], \beta=n_{e} q \alpha, \alpha=\frac{q}{m_{e}\left(v_{e}+j \omega\right)}
$$

where $q$ is the electron charge, $m_{e}$ is the electron mass, $v_{e}$ is the collision frequency, and $\omega$ is the plasma frequency.

The boundary conditions can be written based on the following electron exchange mechanisms near the wall: the loss of electrons due to the departure of the electron flow from the plasma volume, electron amplification due to the secondary and autothermoelectronic emission [1].

Therefore, the equation for the normal component of the electron and energy flux can be written as follows:

$$
\begin{gathered}
\mathbf{n} \cdot \boldsymbol{\Gamma}_{e}=\frac{1-r_{e}}{1+r_{e}}\left(\frac{1}{2} v_{e} n_{e}\right)-\left[\sum_{i} \gamma_{i}\left(\boldsymbol{\Gamma}_{i} \cdot \mathbf{n}\right)+\boldsymbol{\Gamma}_{t} \cdot \mathbf{n}\right] \\
\mathbf{n} \cdot \boldsymbol{\Gamma}_{\varepsilon}=\frac{1-r_{e}}{1+r_{e}}\left(\frac{5}{6} v_{e} n_{\epsilon}\right)-\left[\sum_{i} \gamma_{i} \overline{\varepsilon_{l}}\left(\boldsymbol{\Gamma}_{i} \cdot \mathbf{n}\right)+\overline{\varepsilon_{t}} \boldsymbol{\Gamma}_{t} \cdot \mathbf{n}\right]
\end{gathered}
$$

where $r_{e}$ is the reflection coefficient, $v_{e}$ is the thermal velocity, $\gamma_{i}$ is the secondary emission coefficient, $\Gamma_{t}$ is the thermal emission flux, $\Gamma_{i}$ is the ion flux, $\overline{\varepsilon_{l}}, \overline{\varepsilon_{t}}$ is the mean energy of secondary and auto-thermally emitted electrons.

The equation is solved numerically using the finite element method in the COMSOL Multiphysics software program with an adaptive time step. The total process time was assumed to be $50 \mathrm{~ns}$. The electron density growth proceeds through a stepwise ionization process. The number of required cycles for stepwise ionization varies depending on the plasma density.

\section{Results and discussion}

Figures 1-7 show the results of solving a system of equations. 


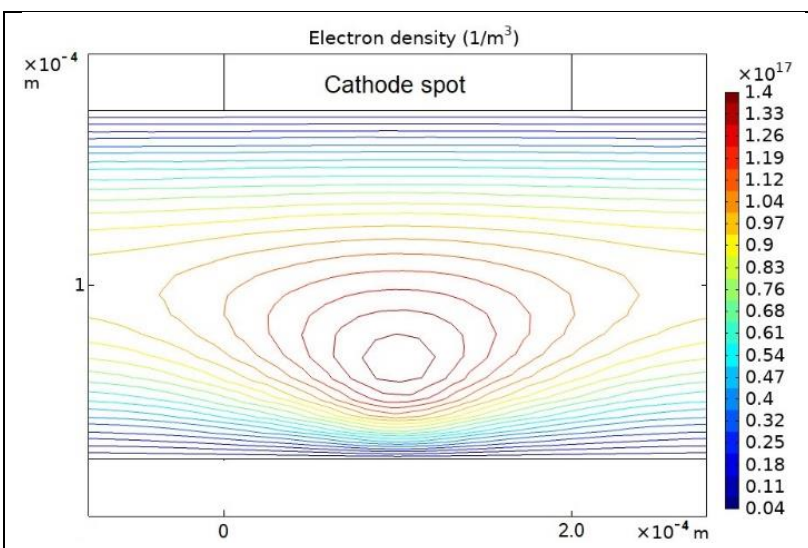

Figure 1. Electron density spatial distribution at the initial stage of streamer nucleation during plasma oscillations in the boundary region.

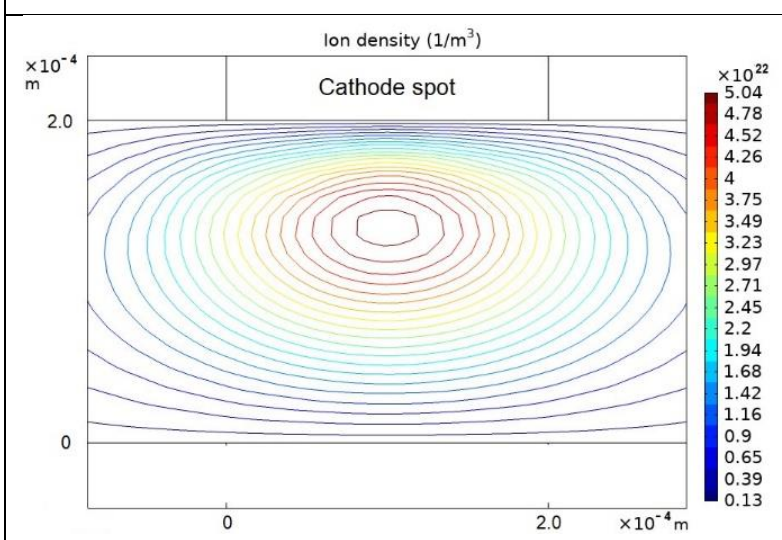

Figure 3 The spatial distribution of the accumulated uncompensated ionic density of the nonequilibrium near-cathode plasma of a vacuum arc.

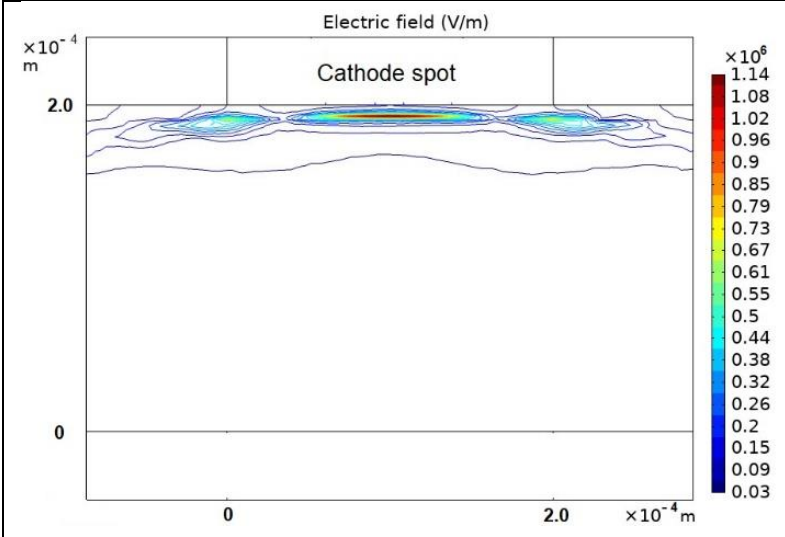

Figure 5. The maximum electric field strength at the cathode-plasma interface. Due to diffusion, the plasma channel decays and three maxima of the electric field are formed.

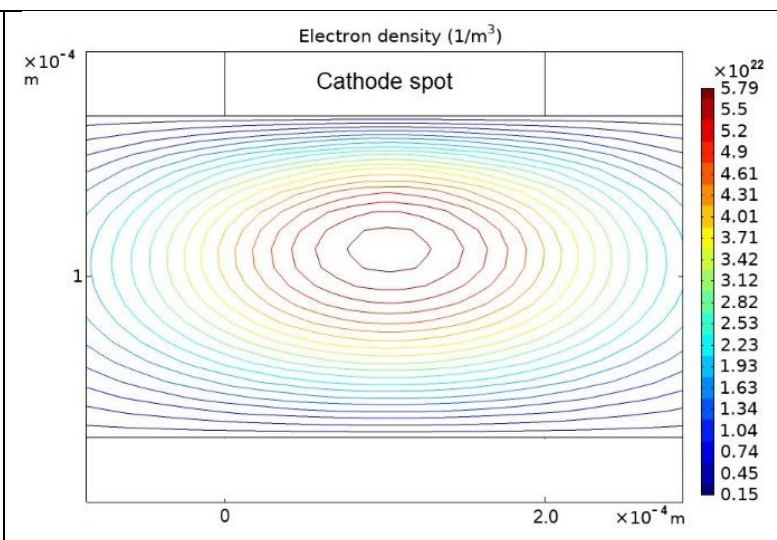

Figure 2. Electron density spatial distribution $50 \mathrm{~ns}$ after the stepwise ionization process.

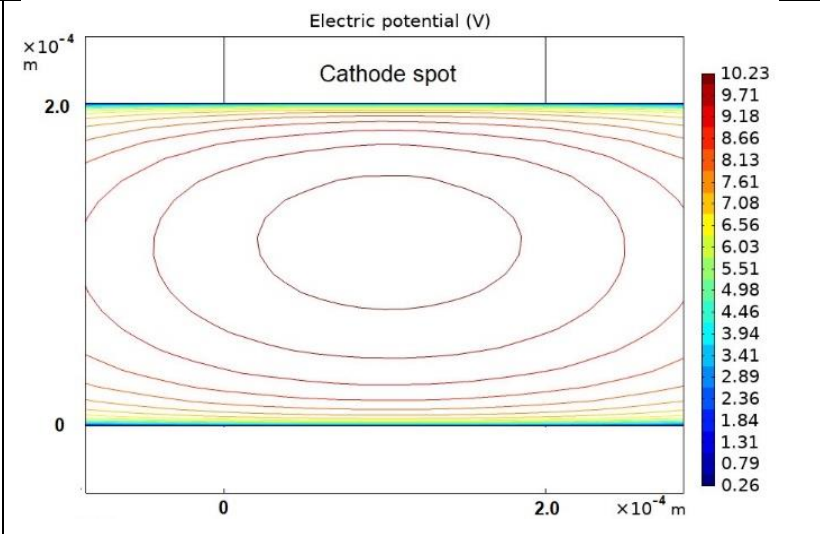

Figure 4. The process of alignment of electron density and the appearance of electric potential.

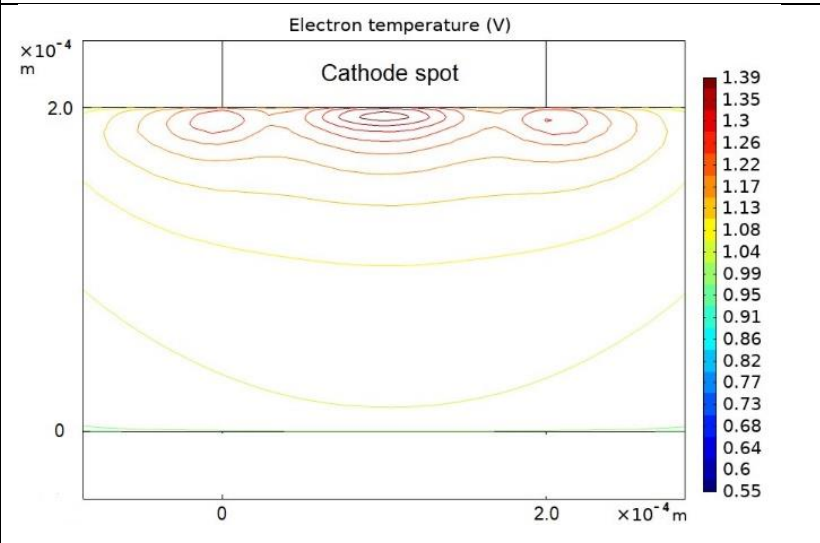

Figure 6. Electron temperature spatial distribution along the plasma column of an arc discharge. 


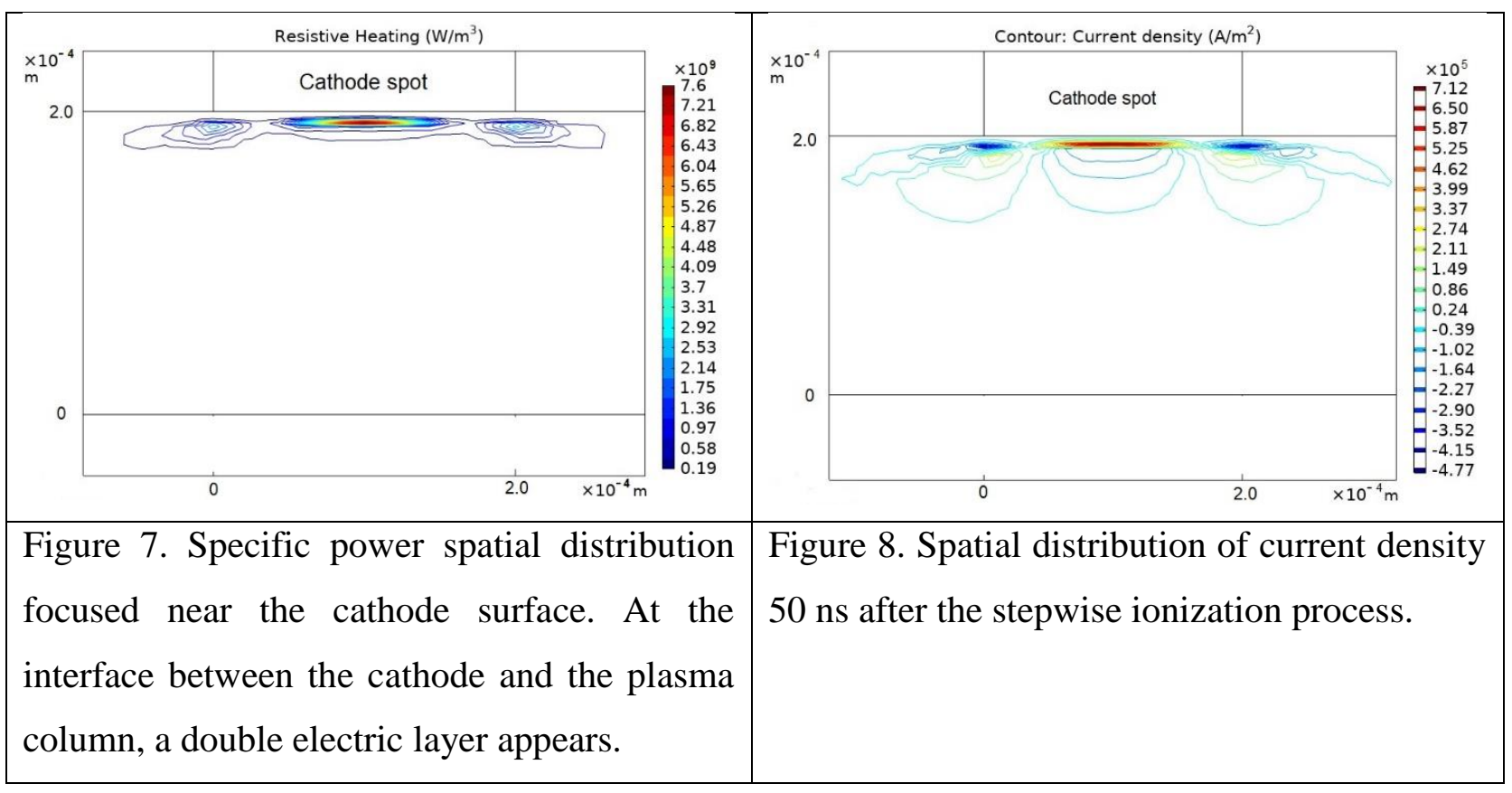

Let's analyze the stages of the ionization process of the cathode spot plasma. Figures 12 show the change in electron density during the wave transition from the plasma edge and the further growth of the positive streamer to the cathode. Under the action of stepwise ionization, the electron density in the cloud gradually increases, and, due to diffusion, the radius of the streamer head increases. At the initial avalanche stage, the bulk charge is very small and the electric field does not change. Due to the influence of diffusion, a part of the electron density moves to the cathode, the central part significantly remains behind. These electrons, due to the high electric field near the cathode and the high ionization frequency, cause a sharp increase in the electron density near the cathode. A new peak is formed, overtaking the previous largest one. But, since the rate of ionization processes is significantly higher than the rate of drift, the electron density peak is smeared. As the plasma density increased, the electron losses enhanced due to diffusion, recombination, and bonds, which reduce the effect of a rapid increase in electron density during step ionization. The recombination processes, the partial departure of electrons towards the anode lead to the equalization of the electron density (Figure 2) and the appearance of an electric potential (Figure 4). The obtained value of the electric potential is in a good agreement with numerous experimental data $[1,2]$ and explains the significant potential drop in the interelectrode space.

The equilibrium value of the potential (Figure 4) is achieved due to many factors: electron inertia, discharge current, applied external voltage, excitation and ionization potential. Resonant regions are formed in the plasma. In these regions, the frequency of atomic excitation is equal to the local plasma frequency. The working range of the local potential is achieved due to the emergence of intense internal currents and a violation of local 
quasineutrality. When the ionization excitation frequency exceeds the plasma frequency, the plasma heating mechanism does not function. The plasma turns into an insulator with low losses and an increase in the external voltage is necessary to maintain the discharge. The authors of [16] experimentally studied the dependence of the argon plasma properties on the frequency of the applied microwave radiation and investigated the conditions of effective heating and plasma losses. It has been shown that a key way to increase the excitationionization frequency is to low the peak electron temperature and to decrease the temperature modulation. Plasma cooling leads to an increase in electron density and a decrease in its escape from the chemical reaction zone. Increasing the frequency of microwave radiation resulted in a rapid increase in the electron density and a decrease in the voltage across the plasma gap. A transition frequency, at which the increase of electron density ceases, and the rate of decrease in the applied voltage decreases, has been found. The transition frequency was determined by the condition $v_{e} \leq v_{e x}$, where $v_{e}$ is the energy dissipation frequency during an electron collision, and $v_{e x}$ is the excitation-ionization frequency in the discharge region. We can talk about the condition of plasma resonance in the discharge gap. In this case, two types of resonance can be distinguished: sequential or boundary plasma resonance and parallel resonance related to volume oscillations of quasi-neutral plasma. In particular, both types of resonance interactions are characteristic of cathode spot plasma. The sequential resonance has a lower frequency and is determined by the thickness $s$ and the length $L$ of the boundary region $\omega_{p} \sim \sqrt{s n_{i} / L}$. For arc discharges, sequential resonance is more efficient concerning to energy consumption because lower temperature and plasma pressure are required. One of the applications of this approach is a very accurate diagnosis of arc plasma properties. In particular, the identification of the frequency of parallel resonance permits the direct determination of the plasma density and electron energy distribution [17].

The accumulated uncompensated ionic density (Figure 3) significantly affects the external electric field, displacing it from the region of increased electron density to the boundary of this region. The region of increased ionization rate also shifts to this region, which leads to an increase in ionic density in front of the boundary. A reflected streamer begins to grow towards the avalanche, displacing the field, and the process repeats. A plasma channel is formed and the field from this channel is displaced to the boundary with the cathode. The region of increased electron density transforms into a plasma state. The maximum electric field is formed at the cathode-plasma interface (Figure 5) and its order of magnitude is in good agreement with the experimental data [18]. Due to diffusion, the plasma channel decays and three maximums of the electric field strength are formed. A high density of seed electrons appears at lateral maximums, which become centers of ionization processes 
and the jump in the cathode spot occurs, as described in [2]. As a result of ionization, additional plasma channels with a high electron density are formed. It should be noted that as a result of stepwise ionization the electron density increases by several orders of magnitude compared to seed electrons. Despite the significant value, the electric field does not cause a significant increase in the electron temperature (Figure 6) in this region, since the wave propagates along the plasma column and the specific energy contribution rapidly decreases with increasing $T_{e}$, the active zone of the discharge is significantly extended. Due to the rapid increase in the radial component of the electric field strength at the boundary of the plasma column, an increase in the electron density drift in the boundary layer is possible. The intensity of the electric field in the plasma determines such an important characteristic for plasma chemistry as the ratio of the temperatures of electrons and heavy particles. The electron temperature, determined both from the ratio of the populations of the upper excited levels of the copper atom and from the intensity of the continuous spectrum, was found to be about $1.3 \mathrm{~V}$, i.e., the plasma in the discharge is far from equilibrium.

The high specific power focused near the cathode surface is of particular interest. Considering the previous discussion and Figures 1-6, the arc discharge can be represented as a cylindrical plasma column with a length of 200 and a diameter of $50 \mu \mathrm{m}$. The power supplied to the discharge excites plasma oscillations at the boundary region. At the interface between the cathode and the plasma column, a double electric layer appears. This layer holds hot electrons in the central zone of the discharge. The presence of a high-temperature zone and a double electric layer at its boundary explains the observed increase in specific power. Figure 7 explains the thermal phenomena on the cathode surface in vacuum arcs, such as inconsistency of the current density with the geometrical dimensions of the cathode spot, high-speed heating of a thin layer of the surface, observed intensities of ultraviolet radiation, high speed of spots moving, similar to the pulsed laser heating, high-speed jets of metal vapor, thermomechanical effects. However, direct experimental confirmation of the results obtained is currently difficult.

Figure 8 shows the spatial distribution of current density in the plasma of the cathode spot of a vacuum arc. Currently, the main criterion for the existence and self-maintenance of the cathode spot of a vacuum arc is the magnitude of the current density. It is the emission current that determines all plasma processes in the cathode spot, and it must be at least $10^{10}$ $\mathrm{A} / \mathrm{m}^{2}$. The value of the density of the emission current is determined experimentally by the method of "autographs" [2] or by studying the spectra. In the model proposed above, the main criterion for the existence of a cathode spot plasma is the plasma frequency, which determines all ionization processes, therefore, the plasma current density is low compared to the emission 
current. The cathode surface can be heated by ion current, electromagnetic radiation, hot plasma electrons, and emission current.

Conclusion. In the present work, the structure of the plasma region of the cathode spot of a low-voltage vacuum arc discharge is calculated within the framework of a computer model. It is shown that due to plasma waves and multistage ionization, a plasma cylindrical channel arises similar to the cathodirectional streamer channel of a spark discharge. As a result of stepwise ionization, the electron concentration increases by several orders of magnitude compared to the initial value. A high field strength near the cathode is formed due to the space charge of slow ions forming a double electric layer. Due to diffusion, the plasma channel decays and three maxima of the electric field are formed. The obtained numerical results are in qualitative and quantitative agreement with the experiments of other authors.

In conclusion, it should be specially noted that the Paschen condition for arc breakdown in the region of the cathode spot must be satisfied and the product of the vapor pressure $(p)$ and the characteristic length of the plasma column $(d)$ can be kept constant even for a plasma gap less than a millimeter in length and a pressure of several atmospheres. Thus, for glow, spark, and arc discharges, the nonequilibrium character of the plasma is a common feature, and the electron temperature can significantly exceed the ion temperature.

Acknowledgments. The work was performed with a support of the grant of the Russian Science Foundation (Project No. 16-19-10054). 


\section{References}

1. Anders A. Cathodic Arcs: From Fractal Spots to Energetic Condensation. New York: Springer, 2008.

2. Kesaev I.G. Cathode processes of an electric arc. Moscow: Science, 1968.

3. Zhang X., Wang L., Jia S., Shmelev D.L. Modeling of Cathode Spot Crater Formation and Development in Vacuum Arc. Journal of Physics D: Applied Physics 50 (2017), 455203.

4. Wang L., Zhang X., Wang Y., Yang Z., Jia S. Simulation of Cathode Spot Crater Formation and Development on CuCr alloy in Vacuum Arc. Physics of Plasmas 25 (2018), 043511.

5. Mesyats G.A., Zubarev N.M. The Rayleigh-Plateau instability and jet formation during the extrusion of liquid metal from craters in a vacuum arc cathode spot. J. Appl. Phys. 117 (2015), 043302. https://doi.org/10.1063/1.4906559

6. Benilov M.S., Cunha M.D., Hartmann W., Kosse S., Lawall A., Wenzel N. Modeling Spots on Composite Copper-Chromium Contacts of Vacuum Arcs and their Stability. IEEE Transactions On Plasma Science 43 (2015), 2253-2260. doi: 10.1109/TPS.2015.2412571.

7. Uschakov A.V., Karpov I.V., Lepeshev A.A., Petrov M.I. Plasma-chemical synthesis of copper oxide nanoparticles in a low-pressure arc discharge. Vacuum 133 (2016) 25-30. http://dx.doi.org/10.1016/j.vacuum.2016.08.007.

8. Karpov I.V., Ushakov A.V., Demin V.G., Shaihadinov A.A., Demchenko A.I., Fedorov L.Yu., Goncharova E.A., Abkaryan A.K. Investigation of the residual stresses effect on the magnetic properties of $\mathrm{CuO}$ nanoparticles synthesized in a low-pressure arc discharge plasma. Journal of Magnetism and Magnetic Materials 490 (2019) 165492. doi: https://doi.org/10.1016/j.jmmm.2019.165492.

9. Lepeshev A.A., Karpov I.V., Ushakov A.V., Nagibin G.E. The morphological and structural features of ferrite compositions $(1-\mathrm{x}) \mathrm{MeFe}_{2} \mathrm{O}_{4} \cdot x \mathrm{P}_{2} \mathrm{O}_{5}$ prepared by plasma spraying. Journal of Alloys and Compounds 663 (2016) 631-635. doi:10.1016/j.jallcom.2015.12.168.

10. Lepeshev A.A., Bayukov O.A., Rozhkova E.A., Karpov I.V., Ushakov A.V., Fedorov L.Yu. Modification of the Phase State and Structure of the $\mathrm{Al}-\mathrm{Cu}-\mathrm{Fe}$ QuasiCrystalline Alloy during Plasma Deposition. Physics of the Solid State 57 (2015) 255-259. doi: 10.1134/S1063783415020249.

11. Karpov I.V., Ushakov A.V., Lepeshev A.A., Fedorov L.Yu. Plasma-Chemical Reactor Based on a Low-Pressure Pulsed Arc Discharge for Synthesis of Nanopowders. Technical Physics 62 (2017) 168-173. doi: 10.1134/S106378421701011X. 
12. Lepeshev A.A., Drokin N.A., Ushakov A.V., Karpov I.V., Fedorov L.Yu., Bachurina E.P. Localization and transfer of charge carriers in $\mathrm{CuO}$ nanopowder by impedance spectroscopy. Journal of Materials Science: Materials in Electronics 29 (2018) 12118-12125. doi: https://doi.org/10.1007/s10854-018-9319-2.

13. Lieberman M.A., Lichtenberg A.J. Principles of Plasma Discharges and Materials Processing // John Wiley \& Sons. New York, 2005. 757 p.

14. Smirnov B.M. Reference data on atomic physics and atomic processes. SpringerVerlag. Berlin, 2008, 173 p.

15. Kee R.J., Coltrin M.E., and Glarborg P., Chemically Reacting Flow: Theory and Practice // Wiley-Interscience. New York, 2003. 928 p.

16. A.R. Hoskinson, J. Gregorio, S. Parsons, and J. Hopwood, Electron confinement and heating in microwave-sustained argon microplasmas. Journal of Applied Physics 117 (2015) 163301. https://doi.org/10.1063/1.4919416.

17. D.D. Blackwell, D.N. Walker, and W.E. Amatucci, Measurement of absolute electron density with a plasma impedance probe. Review of Scientific Instruments 76 (2005) 023503. https://doi.org/10.1063/1.1847608.

18. E.R. Kieft, J.J.A.M. van der Mullen, and G.M.W. Kroesen, V. Banine, K.N. Koshelev, Stark broadening experiments on a vacuum arc discharge in tin vapor. Phys Rev E Stat Nonlin Soft Matter Phys 70 (2004) 066402. doi: 10.1103 / PhysRevE.70.066402. 